\title{
KINOSTERNON INTEGRUM (KINOSTERNIDAE)
}

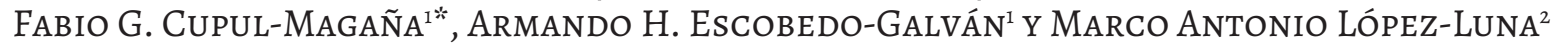

${ }^{1}$ Centro Universitario de la Costa, Universidad de Guadalajara, Av. Universidad 203, Delegación Ixtapa, Puerto Vallarta, Jalisco, 48280, México. ${ }^{2}$ División Académica de Ciencias Biológicas, Universidad Juárez Autónoma de Tabasco, Carr. Villahermosa-Cárdenas km 0.5, Villahermosa, Tabasco, 86039, México.

"Correspondence: fabiocupul@gmail.com

México: Jalisco: Municipio de Puerto Vallarta: Puerto Vallarta: Fraccionamiento Parques Universidad (20.68020 N, -105.21910 W, datum WGS 84, elev. 6 m). 31 de julio 2019. Un macho adulto (longitud del caparazón de $13.5 \mathrm{~cm}$ ) fotografiado por el primer autor próximo a un arroyo en el área urbana de Puerto Vallarta (Fig. 1). Este es el registro más noroccidental de la especie para la costa de Jalisco (Casas-Andreu, 1972; Turtle Taxonomy Working Group, 2017). Se encuentra a $12.5 \mathrm{~km}$ lineales al sur de una localidad del municipio nayarita de Bahía de Banderas, ubicada a $3 \mathrm{~km}$ al suroeste de San Juan de Abajo, $3 \mathrm{~km}$ al este del Valle de Banderas y $3 \mathrm{~km}$ al noreste de San José de Valle [información obtenida de los ejemplares depositados en la colección del National Museum of Natural History, Washington, D.C., USNM 238066 (29 de febrero de 1976) y USNM 238067 (2 de marzo de

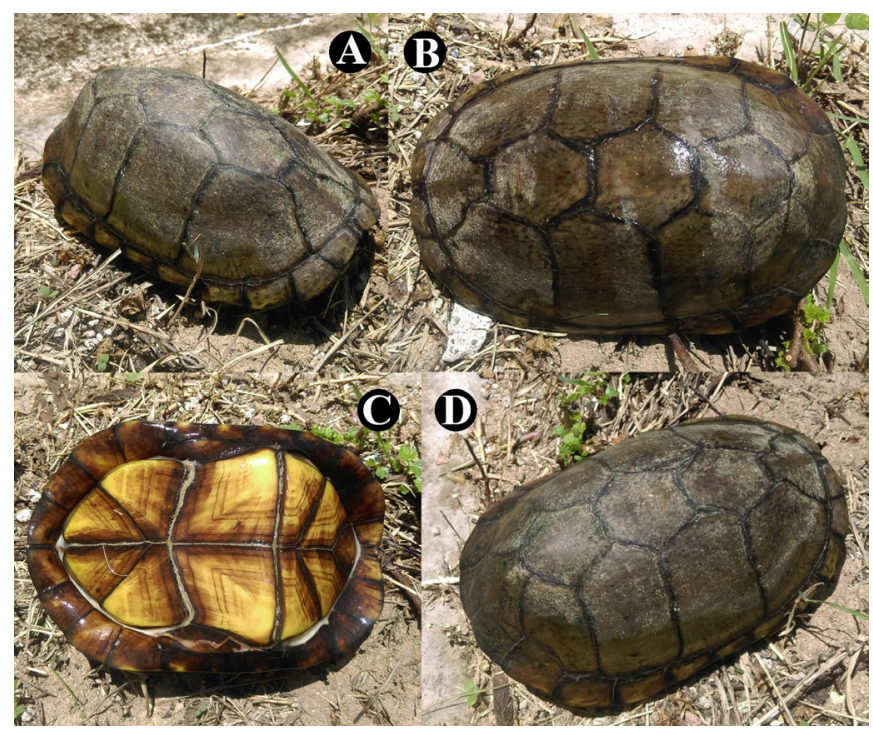

Figure 1. Specimen of Kinosternon integrum observed in Puerto Vallarta, Jalisco (photographic voucher: IBH RF-562). A) Right anterolateral view. B) Dorsal view. C) Ventral view. D) Right posterolateral view. Photographies: Fabio G. Cupul-Magaña.

Figura 1. Ejemplar de Kinosternon integrum observado en Puerto Vallarta, Jalisco (voucher fotográfico: IBH RF-562). A) Vista anterolateral derecha. B) Vista dorsal. C) Vista ventral. D) Vista posterolateral derecha. Fotos: Fabio G. Cupul-Magaña.
1976); VertNet (2019 a, b)], así como a $42 \mathrm{~km}$ lineales al noreste de El Tuito, Jalisco [información obtenida del ejemplar depositado en la Colección Nacional de Anfibio y Reptiles (CNAR) del Instituto de Biología de la Universidad Nacional Autónoma de México (UNAM), IBUNAM CNAR 2680 (1975); Integrated Digitalized Biocollections (2019)]. La información sobre la distribución de la especie para esta región de Jalisco es relevante, ya que en el trabajo de las tortugas de México de Legler \& Vogt (2013) y en la lista anotada de las tortugas del mundo del Turtle Taxonomy Working Group (2017), no se documentan o se omiten localidades para la planicie costera del estado. Una fotografía del ejemplar se depositó en la CNAR del Instituto de Biología de la UNAM (voucher fotográfico IBH RF-562). La identificación se realizó con base en la descripción de Legler \& Vogt (2013) y fue corroborada por Gabriel Barrios Quiroz (Centro de Investigación y Experimentación en Alternativas Agroecológicas S. C.).

Agradecimientos.- A Gabriel Barrios Quiroz, Centro de Investigación y Experimentación en Alternativas Agroecológicas S. C., por la verificación de la identificación de los ejemplares. A Adriana J. González Hernández, CNAR del Instituto de Biología de la Universidad Nacional Autónoma de México, por su apoyo en la asignación de número de catálogo del voucher fotográfico. A los revisores por su comentarios.

\section{LITERATURA CITADA}

Casas-Andreu, G. 1972. Anfibios y reptiles de la costa suroeste del estado de Jalisco, con aspectos sobre su ecología y biogeografía. Tesis de Doctorado, Facultad de Ciencias, Universidad Nacional Autónoma de México, Ciudad de México, México.

Integrated Digitalized Biocollections, 2019. Specimen Record: Kinosternon integrum. https://www.idigbio.org/portal/ records/793ea8d7-c4ab-4799-a639-204c3baf5c51 [Consultado en Agosto 2019]

Legler, J.M. \& R.C. Vogt. 2013. The turtles of Mexico: Land and freshwater forms. University of California Press, Berkeley, USA. 
Turtle Taxonomy Working Group [Rhodin, A.G.J., J.B. Iverson, R. Bour, U. Fritz, A. Georges, H.B. Shaffer \& P.P. van Dijk]. 2017. Turtles of the World: Annotated Checklist and Atlas of Taxonomy, Synonymy, Distribution, and Conservation Status (8th Ed.). En A.G.J. Rhodin, J.B. Iverson, P.P. van Dijk, R.A. Saumure, K.A. Buhlmann, P.C.H. Pritchard \& R.A. Mittermeier (Eds.), Conservation biology of freshwater turtles and tortoises: A compilation project of the IUCN/SSC Tortoise and Freshwater Turtle Specialist Group. Chelonian Research Monographs 7:1292.
VertNet. 2019a. USNM Amphibians \& Reptiles 238066 Kinosternon integrum. http://portal.vertnet.org/o/usnm/amphibiansreptiles?id=http-n2t-net-ark-65665-3be20a829-ef94-47a1-a12e93eb72e1816e [Consultado en Agosto 2019]

VertNet. 2019b. USNM Amphibians \& Reptiles 238067 Kinosternon integrum. http://portal.vertnet.org/o/usnm/amphibiansreptiles?id=http-n2t-net-ark-65665-3f2e64fe8-64c9-4ea6-b404377690299929 [Consultado en Agosto 2019] 